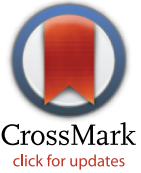

G OPENACCESS

Citation: Yitbarek T, Nega D, Tasew G, Taye B, Desta K (2016) Performance Evaluation of Malaria Microscopists at Defense Health Facilities in Addis Ababa and Its Surrounding Areas, Ethiopia. PLoS ONE 11(11): e0166170. doi:10.1371/journal. pone. 0166170

Editor: Michelle Louise Gatton, Quensland University of Technology, AUSTRALIA

Received: June 19, 2016

Accepted: October 23, 2016

Published: November 28, 2016

Copyright: ๑ 2016 Yitbarek et al. This is an open access article distributed under the terms of the Creative Commons Attribution License, which permits unrestricted use, distribution, and reproduction in any medium, provided the original author and source are credited.

Data Availability Statement: All relevant data are within the paper.

Funding: This work was financially supported by the Addis Ababa University. The funders had no role in study design, data collection and analysis, decision to publish, or preparation of the manuscript.

Competing Interests: The authors have declared that no competing interests exist.

Abbreviations: SPSS, Statistical package for social science; RDT, rapid diagnostic tests; DHMD,
RESEARCH ARTICLE

\section{Performance Evaluation of Malaria} Microscopists at Defense Health Facilities in Addis Ababa and Its Surrounding Areas, Ethiopia

\author{
Tigist Yitbarek $^{1,4 *}$, Desalegn Nega $^{2}$, Geremew Tasew $^{2}$, Bineyam Taye ${ }^{3}$, Kassu Desta ${ }^{4}$ \\ 1 Emanuel Mental Specialized Hospital, Medical Laboratory Service, Addis Ababa, Ethiopia, 2 Malaria, \\ Other Parasite and Vector Borne Parasitic Diseases Research Team; Ethiopian Public Health Institute, Addis \\ Ababa, Ethiopia, 3 Colgate University, Department of Biology, Hamilton, New York, United States of America, \\ 4 Department of Medical Laboratory Sciences, Collage of Health Sciences, Addis Ababa University, Addis \\ Ababa, Ethiopia \\ * tigist.yitbarek@yahoo.com
}

\section{Abstract}

\section{Background}

Blood film microscopy is the gold standard approach for malaria diagnosis, and preferred method for routine patient diagnosis in health facilities. However, the inability of laboratory professionals to correctly detect and identify malaria parasites microscopically leads to an inappropriate administration of anti-malarial drugs to the patients and incorrect findings in research areas. This study was carried out to evaluate the performance of laboratory professionals in malaria diagnosis in health facilities under the Defense Health Main Department in Addis Ababa and its surroundings, Ethiopia.

\section{Method}

A cross sectional study was conducted from June to July 2015. Totally, 60 laboratory professionals out of the selected 16 health facilities were included in the study. Data were collected by distributing standardized pre-validated malaria slide-panels and self-administered questionnaires among professionals, onsite in each study facility. Sensitivity, specificity, and strength of agreement (with kappa score) in performance among the study participants against WHO-certified expert malaria microscopists were calculated.

\section{Result}

Of the 60 study participants, $8.3 \%$ (5/60) correctly read all the distributed slides in terms of parasite detection, species identification and parasite counting; whereas, each of the remaining 55(91.7\%) interpreted at least two slides incorrectly. The overall sensitivity and specificity of participants' performance in detection of malaria parasites were $65.7 \%$ and $100 \%$, respectively. Overall, fair agreement (71.4\%; Kappa: 0.4$)$ in detection of malaria parasite was observed between the study subjects and expert readers. The overall sensitivity and specificity of participants in species identification of malaria parasites were respectively 
Defense Health Main Department; WHO, World health organization; WBCs, White blood cells; $\mathbf{P f}, P$. falciparum; Pv, P.vivax, EQA, external quality assurance; $\mathbf{A A}$, Addis Ababa.
$41.3 \%$ and $100 \%$. Overall, slight agreement (51.1\%; kappa: 0.04 ) in identification of malaria species was observed. Generally, agreement was lower in parasite detection and species identification at low parasite density and mixed infection cases.

\section{Conclusion}

The general agreement between the study participants and expert microscopists in malaria parasite detection and species identification was very low, particularly in the cases of lowparasite density and mixed infections. Therefore, regular external quality assessments and further refreshment trainings are crucial to enhance the skill of professionals in malaria microscopy; particularly for those in non-malarious areas where exposure to malaria diagnosis is low.

\section{Background}

Early diagnosis and prompt treatment are key strategies for the management of malaria to reduce mortality and morbidity [1]. There are different diagnostic methods for malaria case management; the common ones are patient's clinical assessment, microscopic examination of blood films and the use of malaria rapid tests (RDT). Detection of Plasmodium parasites by light microscopy is yet the primary method of malaria diagnosis in most health care facilities throughout the world $[2,3]$. It has long been the method of choice for diagnosis of many parasitic diseases; particularly, it is considered as the gold standard tool for malaria diagnosis still now. However, microscopy requires good technical skills, quality equipments, reagents and a electric power supply $[4,5]$. Thus, the quality of this diagnostic method can easily be affected by one of the above determinants.

Correct anti-malarial therapy is critical to delay emergence of drug resistance, and to avoid wastage of other drugs by incorrect prescription, related to incorrect diagnostic result. Confirmatory diagnosis before treatment initiation recently regained attention, highly influenced by the emerging drug resistance and to save more expensive and not easily affordable drugs in the resource-poor countries $[4,5]$. False positive and false negative malaria diagnostic results produce many consequences up to the death of the patient. In addition, misdiagnosis of malaria results in prescription of inappropriate and high cost drugs for unknown disease, which in turn leads to drug resistance development [6,7].

Low sensitivity and specificity in performance of professionals, in microscopic diagnosis of malaria is a great challenge which has long been described as leading to delayed treatment, development of serious complications, death of patients or exposure to unnecessary treatment [8]. In practice, poor microscopy is a function of multiple factors, including insufficient regular refreshment training for professionals, incorrect blood film preparation techniques, high workload, low quality microscope, and low quality laboratory supplies [9].

Lack of qualified professionals in malaria diagnosis and the lack of regular quality control approaches in the laboratory diagnostic process have been identified as part of possible reasons for low success in malaria control [10]. Many patients are being managed as malaria cases in spite of negative blood film result, which is due to over suspiciousness of health workers or the perceived poor quality of laboratory finding [11].

Compared to malaria endemic areas, the challenge in the quality of malaria microscopy is higher in the health facilities located in the non-malarious areas where laboratory professionals 
have very low exposure in diagnosing malaria cases. Hence, this study was conducted to assess the capacity of professionals in detecting \& identifying malaria parasites on blood film microscopy in the malaria non-endemic Addis Ababa and its surrounding areas, Ethiopia.

\section{Materials and Methods}

\section{Study area and period}

The study was conducted from June to August 2015 in selected health facilities under the Defense Health Main Department (DHMD) of Addis Ababa and its surrounding areas, Ethiopia. Addis Ababa is the capital city of Ethiopia, which is situated in the foothills of the Entoto Mountains and located at an altitude of 2,400 $\mathrm{m}$ above sea level. The area is non-malarious highland fringe; however, as the capital city, it serves as a wide trade center where many migrants are coming to-and-fro as a general. The city is an important administrative center not only for the Federal Government of Ethiopia, but also it is a seat for the African Union, International Organizations and United Nations offices for the whole Africa. Therefore, cases of imported malaria are high in cities of this kind and are usually diagnosed incorrectly for parasite detection and species identification due to the low experience of malaria microscopists. Under the DHMD, there are level-based hospitals such as level-1 hospitals, level-2 hospitals, level-3 hospitals, and the armed force referral teaching hospital. Totally 16 health facilities and on average 4 laboratory professionals per each facility were employed to this study.

\section{Study design and inclusion of study subjects}

A cross sectional study design was used by distributing uniformly prepared Giemsa-stained and unstained malaria blood films from different malaria positive donors. Study subjects were all malaria microscopists in the study health facilities, who were available at the diagnostic facilities during data collection. Professionals who were on leave due to sickness and maternal cases, and non-consenting ones were excluded. Total sample size of 60 malaria microscopists participated on this study.

\section{Data collection process}

Preparation and distribution of malaria slide panels. Based on the patients consent on the study purpose, 5 milliliters of whole blood was collected from each febrile malaria positive patients who attended the national malaria control center in Adama, Ethiopia; which is $99 \mathrm{~km}$ from Addis Ababa. Blood was also collected from malaria negative personnel that had no sign and symptom of malaria and who had no travel history to malaria endemic area. The blood samples were transferred into separate EDTA containing glass tubes.

From the collected blood samples, multiple set of high quality blood film slide panels were prepared; including common malaria parasites ( $P$. falciparum and $P$. vivax), various parasite densities (low, high), mixed infections and negative blood film slides. Each parasite species of low and high density were collected from unique donors. The Parasite densities were reserved for the study based on their direct count from the donors' blood; no laboratory dilution was used to make parasite density lower.

Two WHO-prequalified and certified "level-one" expert microscopists in malaria control center were involved in the preparation and validation of malaria slide panels. Both thick (with $6 \mu \mathrm{l}$ of blood) and thin (with $2 \mu \mathrm{l}$ of blood) films were prepared on a single slide. Each slide with thick and thin film was dried overnight, and the thin film was fixed by dipping in absolute methanol. Then, both thin and thick films were stained with $3 \%$ Giemsa working solution for 30-45 minutes and arranged in sets. The two experts interpreted the films with three 
diagnostic keys: (1) the presence/absence of malaria parasite, (2) identification of parasite species and (3) determining parasite density/ $\mu$ from thick film by counting asexual parasites per 200 leukocytes (or per 500 leukocytes when parasites count was $<10$ ), by assuming a standard WBC count of 8,000 leukocytes/ $\mu$ l.

$$
\text { Parasite } / \mu l=\frac{\text { Number of asexual parasites } \times 8000 \text { leukocytes }}{200 \text { leukocytes }}
$$

Administration of blood films. Totally 12 malaria blood film slide panels, 6 Giemsastained and 6 unstained slides, were administered for assessment of performance in parasites detection, species identification, and quantification. The standardized malaria blood film slide panels were distributed in the following approach: Two negative stained and unstained slides; 2 P. falciparum stained and unstained slides of low density $(140 / \mu \mathrm{l}) ; 2$ P. falciparum stained and unstained slides of high density $(79,640 / \mu \mathrm{l}) ; 2$ P. vivax stained and unstained slides of low density $(440 / \mu \mathrm{l}) ; 2$ P. vivax stained and unstained slides of high density $(54,400 / \mu \mathrm{l}) ; 2$ mixed species $(P f+P v)$ of stained and unstained blood film slides. Blood films were stained with similar Giemsa solution (prepared from similar stock solution and buffer from the central laboratory) by each malaria microscopist under evaluation in all facilities. All study participants used Olympus binocular light microscope equipped with eyepieces (X10) and objectives (x10, x40 and $\mathrm{x} 100$ ), and the same brand immersion oil was used during microscopic examination. Based on WHO recommendation, quantification result of participant was considered correct when it was in between $25 \% \pm$ the mean calculated from result of expert readers. A total of 120 minutes (10 minutes per each slide) was allocated to examine the 12 malaria slides [4].

Data collection tool. Structured questionnaires were used to collect information on the participating facilities and socio-demography of professionals including age, sex, educational status, diagnostic experience, and refreshment trainings. All study participants were formally trained College/University graduated medical laboratory technicians or technologists, and already certified to do malaria microscopy. However, the participants were interviewed for how often they got short-term refreshment trainings aimed to strengthen their skills during their work experience. Such trainings are usually organized by the Ministry of Health $(\mathrm{MOH})$ and/or other nongovernmental organizations (NGO) who are working to support the country health program.

\section{Data analysis}

Data was entered into Microsoft excel sheets and exported to and analyzed using SPSS version 20 for windows. Level of performance in parasite detection, species identification, and quantification was compared with independent demographic parameters. Sensitivity, specificity, and kappa score (to see the strength of an agreement) were calculated to assess performance of laboratory professionals. Based on WHO recommendation, with reference to the percentage agreement in readings between the participants and expert microscopists; malaria microscopists were classified into four:"In-training" ( $<70 \%$ agreement), "Advanced" $(\geq 70 \%-<80 \%)$, "Reference" ( $\geq 80 \%-<90 \%)$, and "Expert" $(\geq 90 \%)$. Kappa value was calculated to see strength of agreement. Based on calculation, the strength was classified as: Kappa of $<0.20$ is slight agreement, $0.21-0.40$ is fair agreements, $0.41-0.60$ is moderate agreement, $0.61-0.80$ is substantial agreement, $0.81-0.99$ is almost perfect agreement. Proportional agreement" [(true positive + true negative)/total)] gives a single number to indicate screening performance. This may be the weighted average of the sensitivity and specificity or the weighted average of the positive predictive value and negative predictive value $[12,13]$. Fisher's exact test was used to 
assess an association between groups and outcomes, for values smaller than 10 in any cell of the data table.

\section{Ethical consideration}

The study was ethically reviewed and approved by the ethical clearance committee of Addis Ababa University. Informed consent forms were signed by the participating health professionals and blood donors. To ensure confidentiality, the participants' data was linked to a code number only.

\section{Result}

\section{Baseline characteristics}

Total of 60 laboratory professionals, who were available in the facility during data collection, participated in the study. The mean age of participants was $32 \pm 5.43$ years and $48(80 \%)$ of participants were males. Regarding educational status, 32(53.3\%) were bachelor degree and 28 (46.7\%) were diploma holders. Majority of the study participants that is $38(63.3 \%)$ had an experience on malaria microscopy for more than two years and 22(36.7\%) had less than two years. Of the total participants, 20(33.3\%) took refreshment training on malaria microscopy at least once. Those individuals who participated in an external quality assurance (EQA) program accounted for 36(60\%) (Table 1).

\section{Performance of laboratory personnel in malaria diagnosis}

Of the 60 study participants, only 5(8.3\%) interpreted all the distributed slides correctly in terms of parasite detection, species identification and parasite counting; whereas, 55(91.7\%),

Table 1. Demography of laboratory professionals under DHMD in Addis Ababa and its surrounding areas, Ethiopia, 2015.

\begin{tabular}{|c|c|c|}
\hline Characteristics & Category & Total study participants $(n=60), \%(n)$ \\
\hline \multicolumn{3}{|l|}{ Age in year } \\
\hline & $20-30$ & $40.0(24)$ \\
\hline & $31-40$ & $46.7(28)$ \\
\hline & $>40$ & $13.3(8)$ \\
\hline \multicolumn{3}{|l|}{ Gender } \\
\hline & Male & $80(48)$ \\
\hline & Female & 20(12) \\
\hline \multicolumn{3}{|c|}{ Educational Status } \\
\hline & Bachelor degree & $53.3(32)$ \\
\hline & Diploma & $46.7(28)$ \\
\hline \multicolumn{3}{|c|}{ Lab participating in EQA } \\
\hline & Yes & $60.0(36)$ \\
\hline & No & $40.0(24)$ \\
\hline \multicolumn{3}{|c|}{ In-service refresher training on malaria microscopy } \\
\hline & Yes & $33.3(20)$ \\
\hline & No & $66.7(40)$ \\
\hline \multicolumn{3}{|c|}{ Frequency of refresher training } \\
\hline & Once & $33.3 \%(20)$ \\
\hline & No & $66.7 \%(40)$ \\
\hline \multicolumn{3}{|c|}{ Experience in routine malaria diagnosis } \\
\hline & $<2$ years & $36.7(22)$ \\
\hline & $\geq 2$ years & 63.3(38) \\
\hline
\end{tabular}

doi:10.1371/journal.pone.0166170.t001 
Table 2. Agreement between malaria microscopists and experts, Addis Ababa, and its surroundings, 2015.

\begin{tabular}{|c|c|c|c|c|c|}
\hline \multicolumn{2}{|l|}{ Parasite Detection } & \multicolumn{2}{|c|}{ Accuracy In Species Identification } & \multicolumn{2}{|c|}{ Quantification within $25 \%$ of true count } \\
\hline Grade & n (\%) & Grade & n (\%) & Grade & $n(\%)$ \\
\hline Expert ( $\geq 90 \%)$ & $5(8.3)$ & Expert ( $\geq 90 \%)$ & $5(8.3)$ & Expert ( $\geq 50 \%)$ & $4(6.7)$ \\
\hline Reference ( $\geq 80 \%)$ & $10(16.7)$ & Reference ( $\geq 80 \%$ ) & 0 & Reference ( $\geq 40 \%)$ & $8(13.3)$ \\
\hline Advanced ( $\geq 70 \%)$ & $5(8.3)$ & Advanced $(\geq 70 \%)$ & $6(10)$ & Advanced ( $\geq 30 \%)$ & $12(20.0)$ \\
\hline In-Training (<70\%) & $40(66.7)$ & In-Training $(<70 \%)$ & $49(81.7)$ & In-Training $(<30 \%)$ & $36(60.0)$ \\
\hline Total & $60(100)$ & Total & $60(100)$ & Total & $60(100)$ \\
\hline
\end{tabular}

Abbreviations: n: number; \%: percent

doi:10.1371/journal.pone.0166170.t002

each of which, interpreted at least two slides incorrectly. Based on WHO grading system, 40 (66.7\%) of participants were at "In-training", five (8.3\%) at "Advanced", $10(16.7 \%)$ at "Reference", and five (8.3\%) at an "Expert level" in malaria parasite detection (Table 2). From the total, 51(85\%) of all participants used not standard quantification system that they reported parasite count in $1+, 2+, 3+$ screening system of quantification. The remaining nine (15\%) counted parasites against 200 or 500 WBCs and determined the total parasites per the standard 8000 WBCs.

A total of 720 blood film slides of which 240 P.falciparum, 240 P.vivax, 120 mixed $P f+P V$, and 120 negative controls were used for evaluation. From the total 720 slides, $514(71.3 \%)$ were correctly reported for the detection of malaria parasites. Of the 600 positive slides ( 60 participants x 10 slides), 248 (41.3\%) on species identification and only 45 slides (7.5\%) on quantification were reported correctly. In the case of negative slides, all of the $120(60 \times 2)$ slides were reported acceptable (Fig 1)

\section{Sensitivity and specificity in performance of microscopists}

Overall reliability. Overall, the sensitivity and specificity of participants' performance in detection of malaria parasites were $65.7 \%$ and $100 \%$, respectively. The overall agreement in

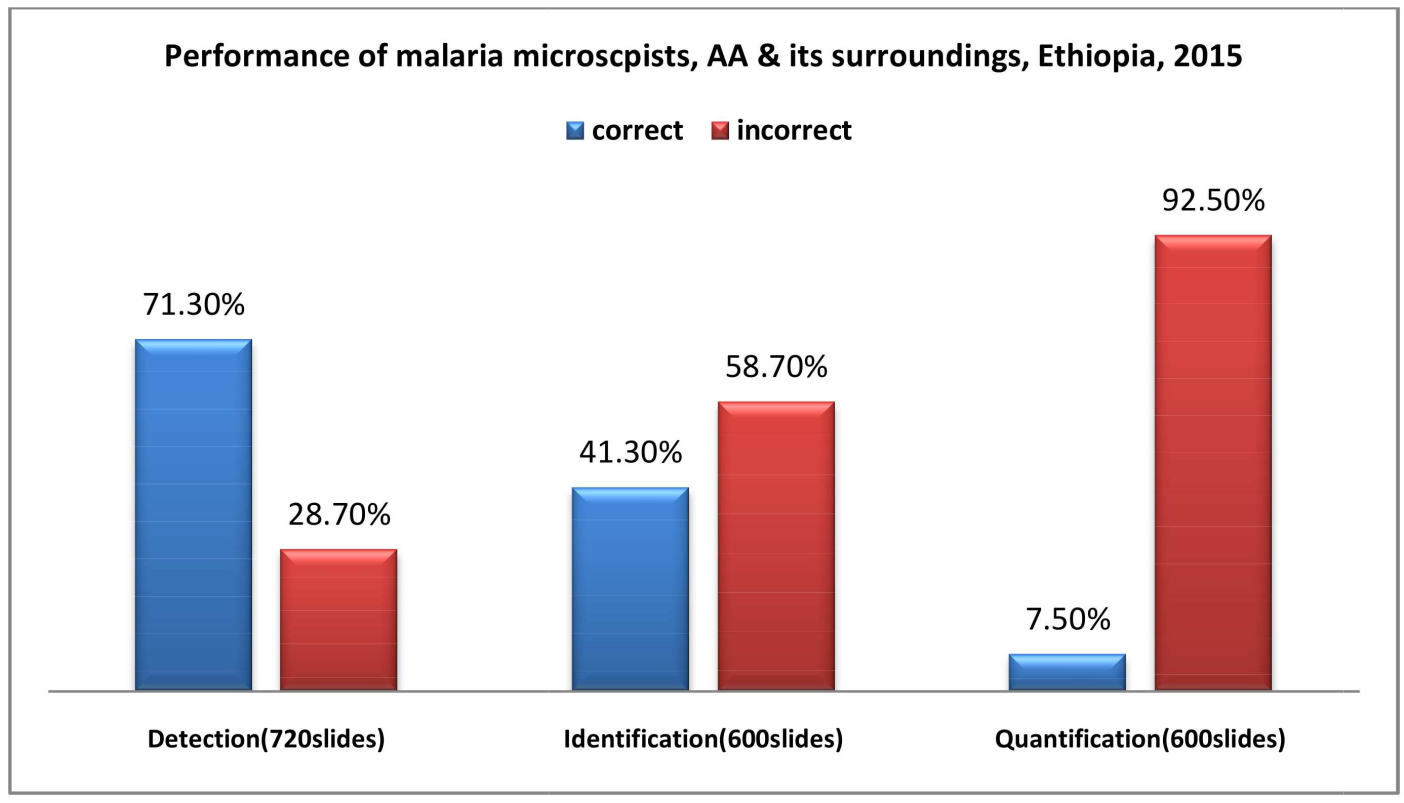

Fig 1. Performance of malaria micrscopists, Addis Ababa, and its surroundings, Ethiopia 2015. doi:10.1371/journal.pone.0166170.g001 
Table 3. Over all sensitivity, specificity and agreement in performance of participants in parasite detection and species identification, Ethiopia, 2015.

\begin{tabular}{|c|c|c|c|c|c|c|c|}
\hline \multicolumn{2}{|l|}{ Participant Reader } & \multicolumn{2}{|c|}{ Expert Reader } & \multirow{3}{*}{\begin{tabular}{|l} 
Sensitivity \\
$65.7 \%$ \\
\end{tabular}} & \multirow{3}{*}{\begin{tabular}{|l|} 
Specificity \\
$100 \%$ \\
\end{tabular}} & \multirow{3}{*}{\begin{tabular}{|r|} 
Agreement \\
$71.4 \%$ \\
\end{tabular}} & \multirow{3}{*}{\begin{tabular}{|r} 
Kappa \\
0.4
\end{tabular}} \\
\hline & & \multirow{2}{*}{\begin{tabular}{|l|} 
Pos \\
394 \\
\end{tabular}} & \multirow{2}{*}{$\begin{array}{l}\text { Neg } \\
0\end{array}$} & & & & \\
\hline Parasite Detection & Pos & & & & & & \\
\hline & $\mathrm{Neg}$ & 206 & 120 & & & & \\
\hline & Total & 600 & 120 & & & & \\
\hline \multirow[t]{3}{*}{ Species Identification } & Correct & 248 & 0 & NA & NA & 51.1 & 0.04 \\
\hline & Incorrect & 352 & 120 & & & & \\
\hline & Total & 600 & 120 & & & & \\
\hline
\end{tabular}

Abbreviation: NA: Not applicable; Pos: positive; Neg: Negative

doi:10.1371/journal.pone.0166170.t003

detection of malaria parasite between the study subjects and expert reader was $71.4 \%$ (Kappa: 0.4 ) which is 'fair agreement' (Table 3). The overall agreement on identification of malaria species was $51.1 \%$ (kappa: 0.04) which is 'slight agreement' (Table 3).

The performance of participants on species identification showed that $45.8 \%$ of blood film slides with P. falciparum, $45 \%$ of slides with P.vivax, and $16.6 \%$ of slides with mixed infections were identified correctly. A total of 100 (83.3\%) of slides with mixed infection were identified wrongly as $P$. falciparum, 97 (40.4\%) of slides with $P$. falciparum were identified wrongly as negative and 109 (45.4\%) of slides with $P$. vivax were identified wrongly as negative (Fig 2).

Of the total 60 participants, the number of participants who scored $<80 \%$ performance was higher among participants who had not taken refreshment training on malaria microscopy (Table 4).

Performance in relation to parasite density and staining of slides prior to the study. Performance in species identification was $75 \%$ and $70 \%$ for high-density $P$. falciparum stained and unstained slides, respectively. In the case of low-density P. falciparum slides, species identification was $25 \%$ for stained and $13.3 \%$ for unstained slides. Overall, the sensitivity and specificity of participants in detection of parasites in the low-density unstained slides were $17.5 \%$

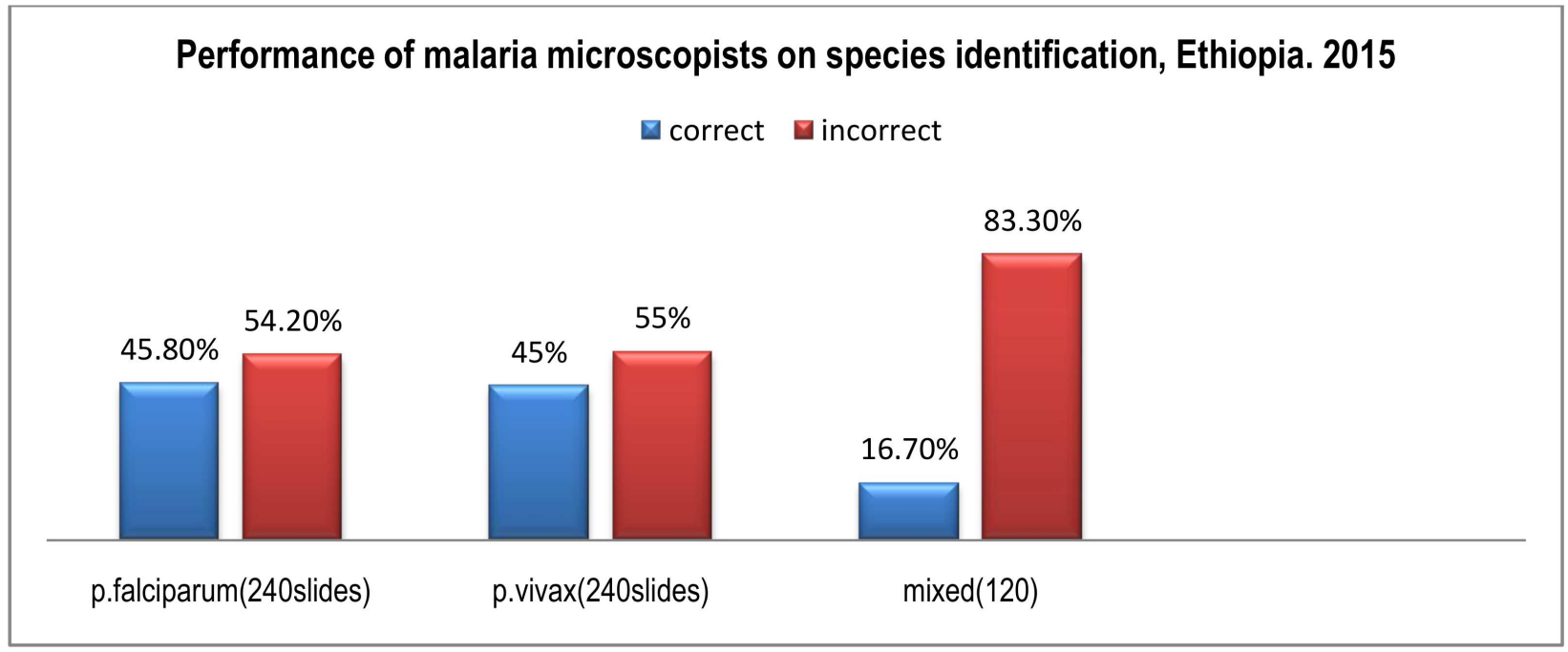

Fig 2. Performance of malaria microscopists in species identification, Ethiopia. 2015.

doi:10.1371/journal.pone.0166170.g002 
Table 4. Demography related to performance score in malaria species identification, Ethiopia, 2015.

\begin{tabular}{l|l|l|l|l}
\hline Variables & Category & $\geq \mathbf{8 0} * *$ & $<\mathbf{8 0} * *$ & Fisher exact test Two-tailed P-value \\
\hline Educational Status & & & & \\
\hline & College Diploma & 2 & 26 & 1.000 \\
\hline Work Experience & University Degree & 3 & 29 & \\
\hline & & & 19 & 0.6998 \\
\hline Malaria microscopy and QA training & $<2$ Years & 3 & 34 & \\
\hline & $\geq 2$ Years & 4 & & 17 \\
\hline & Yes & 3 & 38 & 0.3216 \\
\hline
\end{tabular}

Note:

* stands for 'Passed';

** stands for 'Failed';

QA: Quality Assurance. Fisher's exact test yielded two-tailed P-value is $>5 \%$. The association between rows (groups) and columns (outcomes) is considered to be not statistically significant.

doi:10.1371/journal.pone.0166170.t004

and $100 \%$, respectively. The overall agreement was $58.7 \%$ (Kappa $=0.17)$, which is almost 'slight agreement' (Table 5).

Half of the 720 study blood smears were already stained in the referral laboratory by the expert microscopists and half were unstained before the start of study. Unstained smears were made to be stained by study participants in their laboratories. The participants' performance in both detection and species identification on pre-study stained slides was better than on the blood smears stained by study participants in their own laboratories.

\section{Discussion}

The present study showed poor performance of professionals in malaria microscopy among diagnostic laboratories at defense health facilities in Addis Ababa and its surrounding areas, Ethiopia. Of the 60 study participants, only 5(8.3\%) interpreted all the distributed slides correctly in terms of parasite detection \& species identification; and the rest 55 (91.6\%), each of

Table 5. Overall performance of participants in relation to slide staining and parasite density, Addis Ababa, Ethiopia, 2015.

\begin{tabular}{|c|c|c|c|c|c|c|c|c|}
\hline \multicolumn{2}{|l|}{ Participant Reader } & \multicolumn{3}{|l|}{ Expert Reader } & \multirow{2}{*}{\begin{tabular}{|l|} 
Sensitivity \\
$100 \%$ \\
\end{tabular}} & \multirow{2}{*}{\begin{tabular}{|l} 
Specificity \\
$100 \%$ \\
\end{tabular}} & \multirow{2}{*}{\begin{tabular}{|l|} 
Agreement \\
$100 \%$ \\
\end{tabular}} & \multirow{2}{*}{\begin{tabular}{|l} 
Kappa \\
1 \\
\end{tabular}} \\
\hline Stained high density slide & Reported species & \begin{tabular}{|l|} 
Expected Correct species \\
120 \\
\end{tabular} & \begin{tabular}{|l|} 
Negative \\
0 \\
\end{tabular} & \begin{tabular}{|l|} 
Total \\
120 \\
\end{tabular} & & & & \\
\hline & Negative & 0 & 120 & 120 & & & & \\
\hline & Total & 120 & 120 & 240 & & & & \\
\hline \multirow[t]{3}{*}{ Stained low density slide } & Reported species & 32 & 0 & 32 & $26.7 \%$ & $100 \%$ & $63.3 \%$ & 0.26 \\
\hline & Negative & 88 & 120 & 208 & & & & \\
\hline & Total & 120 & 120 & 240 & & & & \\
\hline \multirow[t]{3}{*}{ Unstained high density slide } & Reported species & 91 & 0 & 91 & $75.8 \%$ & $100 \%$ & $88 \%$ & 0.76 \\
\hline & Negative & 29 & 120 & 149 & & & & \\
\hline & Total & 120 & 120 & 240 & & & & \\
\hline \multirow[t]{3}{*}{ Unstained low density slide } & Reported species & 21 & 0 & 21 & $17.5 \%$ & $100 \%$ & $58.7 \%$ & 0.17 \\
\hline & Negative & 99 & 120 & 219 & & & & \\
\hline & Total & 120 & 120 & 240 & & & & \\
\hline
\end{tabular}

doi:10.1371/journal.pone.0166170.t005 
which, interpreted at least two slides incorrectly. This is in accordance to study finding from Hawassa, Ethiopia [14].

The sensitivity and specificity of the professionals in detecting malaria parasites were $65.7 \%$ and $100 \%$, respectively. The current study showed lower performance in parasite detection when compared to a respective sensitivity and specificity reports of $82 \%$ and $96 \%$ from Hawassa, Ethiopia [14], 88\% and 91\% from Zambia [15], 92\% and 87\% from Uganda [16], and $92 \%$ and $90 \%$ from USA [17]. The lower sensitivity in detection of parasites indicates that there were high false negative results, which implies high misdiagnosis of true infections. The reason for the high specificity (low false positive rate) is presumably related to the small number of true negative samples in the study. As observed in the current study, 97(40.4\%) of P. falciparum slides, and 109(45.4\%) slides with $P$. vivax were identified wrongly as negative. The number of participants who failed to correctly report $P$. falciparum in the current finding was higher than the $27 \%$ failure rate in identification of $P$. falciparum in Canada [11] and in consensus with $39 \%$ failure rate in species identification in a pooled analysis of the 174 slides in USA [18].

The general performance of participants in species identification is lower than in parasite detection in the current study. This matches the findings from other studies [14,18,19]. From the slides with mixed $P$. facliparum and $P$. vivax infections, $100(83.3 \%)$ slides were identified wrongly as P. falciparium mono-infections. This finding is similar to low performance in Hawassa Ethiopia [14], Hong kong [19] and Peruvian Amazon [20]. The possible reasons for this low performance in the current study might be firstly due to the case that study participants were not taking sufficient refreshment training on malaria diagnosis, similar to that reported somewhere else in Ethiopia [21]. Secondly, the study area is less malaria endemic highland area; thus, the professionals had low experience in malaria diagnosis.

In the current study, the overall agreement in detection of malaria parasite between the study subjects and expert readers was $71.4 \%$ (Kappa: 0.4 ), which is categorized as 'fair agreement'. The overall agreement in identification of malaria parasites was $51.1 \%$ (kappa: 0.04 ), which is categorized as 'slight agreement' based on WHO guideline [12,13]. Overall agreement in parasite detection and identification in the current study was lower than the report from malaria endemic areas in Hawassa [14] and Gondar[11], Ethiopia.

In present study, 40(66.7\%) of participants were classified as 'In-training' and 5(8.3\%) were at expert level; which was lower performance than findings from Hawassa where 17(23.6\%) participants were "in-training" and the 18(25\%) were at expert levels [14], and the finding in Indonesia where nearly all microscopists (95.6\%) were at basic or In-training levels, $10(2.3 \%)$ were advanced and $9(2.1 \%)$ were reference microscopists [22]. This difference may be due to the difference in malaria epidemiology in the stated areas.

There were a big difference in parasite detection and species identification in terms of parasite density, and staining of blood film. Performance in species identification was better on high density stained blood film slides (75\%) than on the low density (25\%) stained P. falciparum slides. Performance in species identification was better on high-density unstained (70\%) than on the low density (13.3\%) unstained P. falciparum slides. The study clearly showed the microscopists had low experience in staining of malaria slide smears and had low capacity in malaria diagnosis, particularly in the case of low parasite density. The overall agreement in detection of malaria parasites on low density stained slides was 63.3\% (Kappa: 0.26 ) which is lower than an agreement of $100 \%$ in high density stained slide. The overall agreement on low density unstained slides was $58.7 \%$ (Kappa: 0.17 ) and an agreement on unstained slide with high density was $88 \%$ (kappa: 0.76 ).

The current study brought the first evidence on performance of malaria microscopists in Addis Ababa and its surrounding areas. However, it has come with some limitations, as it 
could not include all malaria microscopists in the study sites; only those who were available during the time of data collection were recruited. The species of malaria parasites used for malaria blood film slide panel preparation were not characterized or molecularly typed by polymerase chain reaction, only identified by certified laboratory technologists.

\section{Conclusion}

The present study showed poor performance of the study participants in malaria microscopy among the diagnostic laboratories at defense health facilities in Addis Ababa and its surrounding areas, Ethiopia. The general agreement between the study participants and expert microscopists in malaria parasite detection and species identification was very low, particularly in the cases of low parasite density and mixed infections. The misinterpretation in malaria diagnosis in turn equally results in misadministration of inappropriate treatment to the patients. This may direct to emergence of drug resistance, exposing patients to suffer from repeated diseases and death, economical loss and wastage of costly drugs. Therefore, to handle these challenges, further regular external quality assessments and trainings are crucial for career competency of professionals, particularly to those who are working in malaria non-endemic areas.

\section{Supporting Information}

\section{S1 File. Study Questionnaire.}

(DOCX)

\section{Acknowledgments}

We would like to thank the Addis Ababa University for financial support \& Defense Health Main Department for allowing us to conduct this study. Our sincere gratitude also goes to data collectors and supervisors for their unreserved support, and the study participants for their participation.

\section{Author Contributions}

\section{Conceptualization: TY KD.}

Data curation: TY DN GT.

Formal analysis: TY DN GT BT KD.

Funding acquisition: TY BT KD.

Investigation: TY DN.

Methodology: TY DN GT BT KD.

Supervision: GT BT KD.

Validation: DN GT BT KD.

Writing - original draft: TY DN.

Writing - review \& editing: GT BT KD.

\section{References}

1. World Health Organization. Bench aids for the diagnosis of malaria infections. Geneva, Switzerland. 2002. 
2. Fernando SD, Ihalamulla RL, Wickremasinghe R, de Silva NL, Thilakarathne JH, Wijeyaratne P, et al. Effects of modifying the World Health Organization standard operating procedures for malaria microscopy to improve surveillance in resource poor settings. Malar J. 2014; 13: 98. doi: 10.1186/1475-287513-98 PMID: 24629093

3. Pierre M, Philippe G, Albert L, Ben A, Simelo K, Jean L, et,al., External quality assessment of malaria microscopy in the Democratic Republic of the Congo. Malar J. 2011; 10: 308. doi: 10.1186/1475-287510-308 PMID: 22008378

4. Haditsch M: Quality and reliability of current malaria diagnostic methods. Travel Med Infect Dis. 2004; 2: 149-160. doi: 10.1016/j.tmaid.2004.03.001 PMID: 17291976

5. Dicko A, Mantel C, Kouriba B, Sagara I, Thera MA, Doumbia S, et al. Season, fever prevalence and pyrogenic threshold for malaria disease definition in an endemic area of Mali. Trop Med Int Heal. 2005; 10: 550-556.

6. Barnish G, Bates I, Iboro J, Newer drug combinations for malaria. BMJ. 2004; 328: 1511-1512. doi: 10. 1136/bmj.328.7455.1511 PMID: 15217846

7. WHO: Malaria Microscopy Quality Assurance, Version 1, WHO, Switzerland, Geneva. 2009.

8. The malERA Consultative Group on Diagnoses and Diagnostics. A research agenda for malaria eradication: Diagnoses and diagnostics. PLoS Med. 2011; 8: e100039617.

9. Wilson ML. Malaria rapid diagnostic tests. Clin Infect Dis. 2012; 54: 1637-1641. doi: 10.1093/cid/cis228 PMID: 22550113

10. Oliveira-Ferreira J, Lacerda MVG, Brasil PP, Ladislau JJLB, Tauil PL, Daniel-Ribeiro CCT. Malaria in Brazil: an overview. Malar J. 2010; 9: 115. doi: 10.1186/1475-2875-9-115 PMID: 20433744

11. Desta KM. Pattern of severe and complicated malaria in children admitted to Gondar Medical College hospital during 1995-2000. [Internet]. Ethiopian Journal of Health Development. 2002. pp. 53-59.

12. Viera AJ, Garrett JM. Understanding interobserver agreement: The kappa statistic. Fam Med. 2005; 37: 360-363. PMID: 15883903

13. Sim J, Wright CC. The kappa statistic in reliability studies: use, interpretation, and sample size requirements. Phys Ther. 2005; 85: 257-68. PMID: 15733050

14. Ayalew F, Tilahun B, Taye B. Performance evaluation of laboratory professionals on malaria microscopy in Hawassa Town, Southern Ethiopia. BMC Res Notes. 2014; 7: 839. doi: 10.1186/1756-0500-7839 PMID: 25422030

15. Barat L, Chipipa J, Kolczak M, Sukwa T. Does the availability of blood slide microscopy for malaria at health centers improve the management of persons with fever in Zambia? Am J Trop Med Hyg. 1999; 60: 1024-1030. PMID: 10403337

16. Msisuka C, Nozaki I, Kakimoto K, Seko M, Ulaya M, Syakantu G. An evaluation of a refresher training intervention for HIV lay counsellors in Chongwe District, Zambia. SAHARA-J J Soc Asp HIV/AIDS 2011; 8: 204-209.

17. Thomson S, Lohmann RC, Crawford L, Dubash R, Richardson H. External quality assessment in the examination of blood films for malarial parasites within Ontario, Canada. Arch Pathol Lab Med. 2000; 124: 57-60. PMID: 10629133

18. Prescott WR, Jordan RG, Grobusch MP, Chinchilli VM, Kleinschmidt I, Borovsky J, et al. Performance of a malaria microscopy image analysis slide reading device. Malar J. 2012; 11: 155. doi: 10.1186/ 1475-2875-11-155 PMID: 22559294

19. Leung WL, Kam KM. Malaria parasite quality assurance program in Hong Kong, 2002-2006. J Hong Kong Inst Med Lab Sci. 11: 8-15.

20. Rosas-Aguirre A, Gamboa D, Rodriguez H, Llanos-Zavalaga F, Aguirre K, Llanos-Cuentas A. [Use of standardized blood smear slide sets for competency assessment in the malaria microscopic diagnosis in the Peruvian Amazon]. Rev Peru Med Exp Salud Publica. 2010; 27: 540-547. PMID: 21308193

21. Mitiku K, Mengistu G, Gelaw B. The reliability of blood film examination for malaria at the peripheral health unit. EthiopJHealth Dev. 2003; 17: 197-204.

22. Ekawati LL, Herdiana H, Sumiwi ME, Barussanah C, Ainun C, Sabri S, et al. A comprehensive assessment of the malaria microscopy system of Aceh, Indonesia, in preparation for malaria elimination. Malar J. 2015; 14: 240. doi: 10.1186/s12936-015-0746-8 PMID: 26062808 